Kang, H., Cheng, P., Yu, Z., and Zheng, H. (2014) A two-stage traveling-wave thermoacoustic electric generator with loudspeakers as alternators. Applied Energy, 137 . pp. 9-17. ISSN 0306-2619.

Copyright (C) 2014 Elsevier Ltd.

A copy can be downloaded for personal non-commercial research or study, without prior permission or charge

Content must not be changed in any way or reproduced in any format or medium without the formal permission of the copyright holder(s)

When referring to this work, full bibliographic details must be given

http://eprints.gla.ac.uk/97982

Deposited on: 17 November 2014

Enlighten - Research publications by members of the University of Glasgow http://eprints.gla.ac.uk 


\title{
A two-stage traveling-wave thermoacoustic electric generator with loudspeakers as alternators
}

\author{
Huifang K ang ${ }^{1} *$, Peng Cheng ${ }^{1}$, Zhibin Y ${ }^{2}{ }^{2}$, Hongfei Zheng ${ }^{1}$
}

${ }^{1}$ B eijing Institute of Technology, B eijing, China, 100081

${ }^{2}$ School of Engineering, U niversity of Glasgow, Glasgow, Scotland, UK, G $128 Q \mathrm{Q}$

${ }^{*}$ Corresponding author: Huifang K ang

E-mail: kanghf@bit.edu.cn; $\quad$ Tel.: +86-10-68914304 Fax: +86-10-68949859 


\section{ABSTRACT}

This paper presents the design, construction and tests of a traveling-wave thermoacoustic electric generator. A two-stage travelling-wave thermoacoustic engine converts thermal energy to acoustic power. Two low-impedance linear alternators (i.e., audio loudspeakers) were installed to extract and convert the engine's acoustic power to electricity. The coupling mechanism between the thermoacoustic engine and alternators has been systematically studied numerically and experimentally, hence the optimal locations for installing the linear alternators were identified to maximize the electric power output and/or the thermal-to-electric conversion efficiency. A ball valve was used in the loop to partly correct the acoustic field that was altered by manufacturing errors. A prototype was built based on this new concept, which used pressurized helium at 1.8 MPa as the working gas and operated at a frequency of about $171 \mathrm{~Hz}$. In the experiment, a maximum electric power of $204 \mathrm{~W}$ when the hot end temperature of the two regenerators reaches $512^{\circ} \mathrm{C}$ and $452^{\circ} \mathrm{C}$, respectively. $\mathrm{A}$ maximum thermal-to-electric efficiency of $3.43 \%$ was achieved when the hot end temperature of the two regenerators reaches $597^{\circ} \mathrm{C}$ and $511^{\circ} \mathrm{C}$, respectively. The research results presented in this paper demonstrate that multi-stage travelling-wave thermoacoustic electricity generator has a great potential for developing inexpensive electric generators.

Key words: Thermoacoustic; Electricity generator; loudspeaker; Travelling-wave 


\section{Introduction}

Due to the concerns about the depletion of fossil fuels and global warming, the utilization of low temperature heat sources, such as industrial waste heat sources and solar energy, has drawn enormous attention. Different technologies have been developed for energy recovery application at different scales. Several advanced thermodynamic cycles such as the Stirling cycle, Organic Rankine cycle, Kalina cycle, U ehara cycle, and triangle cycle have also been developed in order to generate power from relatively low temperature heat sources. Organic Rankine cycle (ORC) systems that use organic fluid with a low boiling point as the working medium and a turbine as expander have demonstrated a great potential to generate power from waste heat sources at medium and large scale (i.e. $10 \mathrm{~kW}$ to several MW). There are also research efforts to develop small-scale ORC systems to generate around $1 \mathrm{~kW}$ to meet the energy demand of households, which has not been very successful so far due to the lack of suitable expanders.

Stirling engine is considered as a promising technology for generating power from waste heat source or solar energy at a relatively small scale. However, conventional Stirling engines usually rely on the clearance sealing at high temperature, and thus require high manufacturing costs. Travelling-wave thermoacoustic engine is essentially the acoustic equivalent of the conventional Stirling engine. It uses a compact acoustic network to obtain the proper time phasing within a regenerator to achieve a Stirling-like thermodynamic cycle to convert the thermal energy to acoustic energy. A thermoacoustic engine usually consists of several simple parts such as an 
acoustic resonator, heat exchangers, and a section of porous materials called a regenerator, and thus eliminates mechanical moving components. Therefore, thermoacoustic engines are simple, reliable, and almost free of maintenance [1-8]. However, they also have some drawbacks. Their power density is relatively low as they usually require a large size acoustic resonator. Their efficiency is low er than the conventional Stiring engine or internal combustion engines.

Due to the aforementioned unique features, travelling-wave thermoacoustic engines have attracted a lot of attention in the past decades. Various prototypes based on different concepts have been constructed, tested and reported [1-4]. So far, the most efficient traveling-wave thermoacoustic engine [1] delivered $710 \mathrm{~W}$ to its resonator with an efficiency of 0.30 which corresponds to $41 \%$ of the Carnot efficiency, when the hot end temperature of regenerator reaches $725^{\circ} \mathrm{C}$. A dditionally, it uses 30-bar helium as working gas and the frequency of is $80 \mathrm{~Hz}$. It has a quarter wave-length acoustical resonator that is around 4 meters in length.

Linear alternators with flexure bearings and clearance seals can be used to convert thermoacoustic engine acoustic power to electricity, which can achieve a transduction efficiency up to $90 \%[9,10]$. A thermoacoustic generator combines the advantages of a thermoacoustic engine and a linear alternator to generate electricity from low temperature heat sources with high efficiency and high reliability. There have been continuous research efforts to develop travelling-wave thermoacoustic electric generator in the past decade [11-17]. However, the linear alternators are costly, which counteracts the advantages of thermoacoustic engines such as simplicity and 
low cost. To pave the way for a possible commercialization, the thermoacoustic electric generator based on linear alternator will be in head-to-head competition with the generators based on conventional Stirling engines which have been on the market for a while. Hence, there is a need to develop inexpensive linear alternators to facilitate the development of inexpensive and reliable thermoacoustic generators to meet the fast growing waste heat recovery market. To meet these challenges, research efforts have been made to develop inexpensive linear alternators using conventional audio loudspeaker technologies [3, 18].

The audio loudspeakers usually have low acoustic impedance when they are coupled to thermoacoustic engines, which bring new design challenges and considerations. Yu et al. systematically investigated these issues theoretically and experimentally [3]. A low-cost thermoacoustic electric generator prototype was developed by placing an audio loudspeaker in the looped-tube traveling-wave thermoacoustic engine as an alternator [3, 18]. Phase tuning techniques (i.e. tuning stub and phase shifter) were developed to correct the acoustic field after the alternator was introduced to the engine. The inexpensive prototype generator using atmospheric air as working gas and a six-inch $B \& C$ subwoofer as alternator, produced $11.6 \mathrm{~W}$ of electrical power. This shows the potential for developing inexpensive electricity generators for energy recovery from waste heat sources based on thermoacoustic technology. Later, a combustion driven prototype was also developed to fully demonstrate the development of low cost thermoacoustic electricity generators for rural communities that are off the national grid [20, 21]. Chen et al. [22] also reported 
their development of low cost two-stage travelling-wave thermoacoustic generators to generate electricity from waste heat energy of cooking stoves. The propane stove driven thermoacoustic generator produced approximately 15 Watts of electricity using an audio loudspeaker as the alternator. A wood-burning stove powered thermoacoustic generator was constructed and tested, which generated 22.7 watts of electricity [23].

This research continues the effort on the development of inexpensive thermoacoustic electric generators. In order to improve the power density, two stage engines and two alternators are designed in one loop. The modeling and simulation of the system, together with the experimental results, are presented and discussed in this paper. The goal of this research is to further demonstrate the potential of low cost thermoacoustic generator, and ultimately push forward the development of inexpensive thermoacoustic generators.

\section{Selecting a loudspeaker as alternator}

The modelling and analysis for using loudspeakers as linear alternators have been described and presented in detail in the reference [19]. The loudspeaker can be simplified and schematically represented by the elements as shown in Fig. 1 of reference [19]. The diaphragm has an area of $S$. The total moving mass is $M_{m}$. The stiffness of the suspension system is $K_{m}$. The mechanical resistance is $R_{m}$. The coil has an inductance $L_{e}$ and resistance $R_{e}$. The force factor is $B I$, and the electric load has a resistance $R_{L}$. The electrical power extracted by the load resistor can be written as [19], 


$$
P_{e}=\frac{1}{2} \frac{\left|U_{1}\right|^{2}}{S^{2}} \frac{(B I)^{2} R_{L}}{\left(R_{e}+R_{L}\right)^{2}}
$$

Electric power output reaches the maximum when $R_{L}=R_{e}$,

$$
P_{e, \max }=\left.\left.\frac{1}{8}||\right|_{1}\right|^{2} \frac{(B I)^{2}}{S^{2} R_{e}}=\frac{1}{8}\left|U_{1}\right|^{2} \frac{(B I)^{2}}{S^{2} R_{L}} .
$$

Equation (2) can estimate the maximum electric output of a given alternator, and therefore is a performance indicator for selecting a suitable loudspeaker as the alternator. According to equation (2), the maximum electric power output is proportional to $\left|U_{1}\right|^{2}$ and $(B I)^{2} / S^{2} R_{L}$. The volumetric velocity $U_{1}$ is determined by the engine parameters and operating conditions, and is limited to certain value for a given engine. $(B I)^{2} / S^{2} R_{e}$ (or $(B I)^{2} / S^{2} R_{L}$ ) is the acoustic impedance of the loudspeaker. It can be found that this acoustic impedance should be increased to increase the electric power output for a given volumetric velocity. A nalogously to electric networks, for a given current, the power consumed by a resistor can be increased by increasing its resistance.

On the other hand, for the loudspeaker tested in this paper, $E$ E $L_{e}$ is much less than $R_{e}$ or $R_{L}$. The inductance of the coil is negligible, and therefore the efficiency can be approximately written as [19]

$$
\eta=\frac{(B \mid)^{2} R_{L}}{R_{m}\left(R_{e}+R_{L}\right)^{2}+(B I)^{2}\left(R_{e}+R_{L}\right)} .
$$

When load resistance reaches

$$
R_{L}=R_{e} \sqrt{1+\frac{(B I)^{2}}{R_{m} R_{e}}} .
$$

The transduction efficiency reaches its maximum as [19] 


$$
\eta_{e, \max }=\frac{(B \mid)^{2} \sqrt{1+\frac{(B I)^{2}}{R_{m} R_{e}}}}{R_{m} R_{e}\left(1+\sqrt{1+\frac{(B I)^{2}}{R_{m} R_{e}}}\right)^{2}+(B \mid)^{2}\left(R_{e}+\sqrt{1+\frac{(B I)^{2}}{R_{m} R_{e}}}\right)} .
$$

Both maximum electric power output and the maximum transduction efficiency are useful performance indicators to evaluate and select loudspeakers as alternators. Table 1 shows the comparison among 14 loudspeakers based on the aforementioned performance indicators. The frequency is fixed at $171 \mathrm{~Hz}$, which is the design frequency of the thermoacoustic generator presented in this paper.

According to Table 1, one can see that, for the given frequency, the listed loudspeakers have transduction efficiencies in the range $18.5-58 \%$. The acoustic impedances, $(B I)^{2} / S^{2} R_{L}$, are quite different from one to another. Loudspeaker $B \& C$ $8 \mathrm{NW} 51$ has the highest acoustic impedances of $94713 \mathrm{~N} \cdot \mathrm{s} / \mathrm{m}^{3}$. According to equation (2), this loudspeaker can potentially produce the maximum electric power for a given volumetric velocity. Therefore, it is selected to construct the thermoacoustic generator presented in this paper.

Furthermore, the maximum electric power can al so be expressed as,

$$
P_{e, \max }=\frac{1}{8} \frac{(B I)^{2} x_{\max }^{2}}{r} \omega^{2}
$$

According to Eq. (6), the maximum electric power is proportional to $\omega^{2}$. The speed of sound in helium is greater than that in air, which leads to a higher frequency with helium as working fluid. Thus, the electric power can be higher by using helium as working gas than for air. Additionally, helium has a smaller Prandtl number $\sigma=0.679$ and the larger ratio of specific heats $\gamma=1.67$, which are useful to 
improve the thermoacoustic engine efficiency [24, 25]. Thus, this thermoacoustic system uses helium as the working gas.

\section{Concept design}

The transduction efficiency of an alternator is independent from the acoustic field within the thermoacoustic engine, and is only determined by its own electrical and mechanical parameters. For this reason, the design of the whole system (i.e. thermoacoustic generator) should focus on optimising the acoustic field within the thermoacoustic engine to maximising the thermodynamic conversion efficiency and minimising the losses due to the acoustic power transmission.

There are two ways to couple an alternator to the looped-tube engine, one is to install the alternator within the loop (denoted as Loop-A Iternator hereafter) [18, 25], and the other is to install the alternator through a branched tube (denoted as Branch-A lternator hereafter) [26]. These two coupling mechanisms both have pros and cons. The Loop-A Iternator can eliminate the Gedeon streaming within the looped engine, and thus reduces the heat losses from the hot heat exchangers. However, the L oop-A Iternator causes large pressure drops and thus sharp acoustic impedance drops when acoustic waves propagate through the diaphragm. A branch stub is usually required to compensate for this effect $[3,18]$. The B ranch-A Iternator is coupled to the looped engine through a branched pipe with a length around a quarter of the acoustic wave length [26]. The opening of the pipe to the engine is tuned to high acoustic impedance so that it would not affect the looped-tube engine significantly. However, 
the looped-tube engine still requires extra measures, such as a membrane or jet-pump, to supress the Gedeon streaming [1].

In the looped-tube travelling-wave thermoacoustic engine, there are usually two high impedance regions and two low impedance zones. The engine stages should be located in the high impedance region to avoid acoustic losses. According to equation (2), it can be found that the electric power output is proportional to $\left|U_{1}\right|^{2}$ for a given alternator. Therefore, the alternators in this paper should be installed in low impedance regions to obtain a relatively high volumetric velocity. Thus two stage traveling wave engines and two alternators were arranged in a looped-tube to effective utilise the space.

Following the design philosophy discussed above, after a series of careful trade-off, a new configuration is proposed as follows: Two engine stages are located at high impedance regions, and an alternator is installed in the engine loop in a low impedance zone. Its hermetic diaphragm suppresses the Gedeon streaming along the engine. The other alternator is installed through a branched pipe which is carefully designed to provide a phase tuning effect to correct the engine's acoustic field, which is altered by the alternator within the engine.

In practical thermoacoustic systems, there is neither pure travelling-wave mode nor pure standing-wave mode. The thermoacoustic effect is the result of the combined interaction of the travelling-wave component (TWC) and the standing-wave component (SWC) in the thermoacoustic system [27-29]. In a thermoacoustic engine, the generated acoustic energy can be transferred by the TW C or stored as the SWC. In 
the thermoacoustic cooler, the consumed acoustic energy can be supplied by the TWC or compensated from the acoustic energy stored in the SWC. In the linear-type thermoacoustic system, the acoustic energy is stored as SW C, and transferred by the TW C. The interaction between acoustic energy storage and transmission stabilises the acoustic field in a thermoacoustic system. For linear engine configuration, there is an obvious terminal boundary condition, so it is easy to determine the acoustic field distribution [30]. In the looped-tube type thermoacoustic system, the traveling wave can also store the acoustic energy due to the closed-loop acoustical boundary conditions. Thus, in the loop, the acoustic energy can be stored by both TWC and SWC, which make the acoustic field very complicated [31-33]. Therefore, manufacturing errors may significantly affect the acoustic field. In present thermoacoustic generators, a ball valve is used in the loop to partly correct the acoustic field that was altered by the manufacturing errors.

Fig. 1 schematically shows the thermoacoustic generator prototype presented in this paper. There are two engine stages, TAE1 and TAE2. Each engine stage has a main ambient heat exchanger (MAHE), a regenerator (REG), a hot heat exchanger $(H H X)$, a thermal buffer tube (TBT), and a secondary ambient heat exchanger (SAHE). There are two alternators, Alternator1 and Alternator2. Alternator1 is installed through a branched pipe, while Alternator2 is installed within the loop between two engine stages.

\section{Numerical simulations}


The concept developed as shown above provides useful design principles and guidelines to optimise this complicated system. However, in order to further design the optimal dimensions of the components and operating parameters for the whole system, more advanced quantitative modelling and simulations are required. DeltaEC [22] (Design Environment for Low-amplitude ThermoA coustic Energy Conversion) developed based on linear thermoacoustic theory has been validated as a robust design tool in the past decades, and therefore was employed to design and optimise the thermoacoustic generator presented in this paper. The design involves the searching for optimal values of a large number of parameters and dimensions. The target is to find the best combination of them. Furthermore, compromises had to be made due to manufacturability and availability of some parts. Such optimization processes are tedious and vey time consuming, and therefore are impossible to be presented in detail due to the limited length of this paper. The simulation results presented in this paper represent the actual prototype that was constructed based on the optimized DeltaEC model, which therefore allows us to compare the experimental results and the theoretical predictions.

The size of a thermoacoustic engine/generator is determined by the operating frequency or vice versa, as it has to operate on the natural acoustic mode defined by the acoustic boundary conditions. Trade-off has been made to achieve different design goals including high efficiency, compact size, and availability of alternator. Therefore, the frequency is selected to be about 171 , leading to a total length of about 5.75 meters. This thermoacoustic generator uses pressurized helium at $1.8 \mathrm{MPa}$ as the 
working gas, and operates at a frequency of $171 \mathrm{~Hz}$. For safety, the hot end temperature of the TAE's regenerator should be kept around $600{ }^{\circ} \mathrm{C}$ or below due to the limited material strength. Thus, in the simulation, the temperature of the two hot heat exchangers is $850 \mathrm{~K}$, and the temperature of the ambient heat exchangers is 300 $\mathrm{K}$. The dimensions and geometries of each component are determined through a systematical optimization processes and are given in Section 5 in detail. The calculation results of the acoustic field in the system are shown in Fig. 2 to demonstrate the implementation of the design philosophy.

Figure 2a shows the pressure amplitude distribution along the system. There are two maxima and two minima of pressure amplitude along the loop and three sharp pressure drops. The pressure drop is caused by the resistance of the two regenerators and Alternator2. Fig. $2 \mathrm{~b}$ shows the distribution of volumetric velocity along the system. There are also two maxima and two minima along the loop. The two minima of the volumetric velocity are at the cold end of the regenerator. The small volumetric velocity within the regenerator is preferred to avoid high viscous dissipation. The two alternators are located in the near maxima of the volumetric velocity, which is better for generating electric power according to equation (2). It can also be seen that there is a sudden decrease of the volumetric velocity at the location of the stub.

Fig. 2c shows the acoustic impedance along the system. It can be seen that the acoustic impedance is the highest at the cold end of the regenerators hence the acoustic losses can be minimized. The stub introduces a sudden increase of the acoustic impedance along the loop. This is because the pressure amplitude at the stub 
junction is constant, while the volumetric velocity has been shunted partly to the stub. From Fig. $2 c$, one can also find that the alternator is very close to the minimum of acoustic impedance. This is also a design feature of the device to obtain a sufficient volumetric velocity to drive the alternator to the maximum excursion, which subsequently maximizes the electrical power output.

Fig. $2 \mathrm{~d}$ shows the phase difference between pressure and velocity oscillations along the system. It can be found that the two regenerators work in the region of $-50^{\circ}<\varphi_{z}<-10^{\circ}$ and $-2^{\circ}<\varphi_{z}<33^{\circ}$. It is found that $\left|\varphi_{z}\right|$ increases sharply in the alternators, and close to $90^{\circ}$. This is due to the fact that the acoustic power is extracted in the alternator and so the traveling wave component drops sharply [27].

Fig. 2e shows the acoustic power flow along the system. It can be found that around 483W of acoustic power flows into the cold heat exchanger of TAE 1 where it dissipates around $36.4 \mathrm{~W}$. The remaining $447.0 \mathrm{~W}$ then flows into the cold end of the regenerator of TAE1. Within the regenerator, the acoustic power is amplified to around $882.2 \mathrm{~W}$. These calculation data show that the TAE1 can produce a net acoustic power of about $435.2 \mathrm{~W}$ at an input heat power of $1057 \mathrm{~W}$, corresponding to a thermodynamic conversion efficiency of $41.2 \%$. A fter being dissipated in the hot heat exchanger, thermal buffer tube, the secondary ambient heat exchanger and the resonator, the residual acoustic power of about $774.9 \mathrm{~W}$ is then divided into two parts at the T-junction: one part of about $218.5 \mathrm{~W}$ flows into the stub and the other part of $556.4 \mathrm{~W}$ continuously flows in the loop. In the stub, the Alternatorl extracts about 203.7 W of acoustic power. In this simulation, the alternator produces $92.7 \mathrm{~W}$ of 
electricity. Therefore, the calculated acoustic-to-electric efficiency is about $45.5 \%$. In the loop, 504.4W of acoustic power is fed into the cold end of the regenerator of TAE2. Within the regenerator, the acoustic power is amplified to around $918.1 \mathrm{~W}$. The calculation data show that the TAE2 produces a net acoustic power of about $413.8 \mathrm{~W}$ with an input heat power of $1208 \mathrm{~W}$, corresponding to a thermodynamic conversion efficiency of $34.2 \%$. Then, $850.3 \mathrm{~W}$ of acoustic power is fed into Alternator 2 which extracts about $338.5 \mathrm{~W}$ of acoustic power. In this simulation, A lternator2 generates $153.9 \mathrm{~W}$ of electricity. Therefore, the calculated acoustic-to-electric efficiency also is 45.5\%. As mentioned above, the total electric power generated from the system is $246.6 \mathrm{~W}$, and the total thermal-to-electric efficiency is $10.9 \%$.

The total thermal-to-electric efficiency is $10.9 \%$, which is indeed much lower that the reported $34.3 \%$ in Ref [11] where a linear alternator was used as the transducer and mechanical resonator. In the current design, there are two reasons leading to lower efficiency compared with that system in Ref [11]. Firstly, compared with using linear alternator as mechanical resonator, the long acoustic resonator dissipated considerable acoustic power. Secondly, the loudspeaker has much lower transduction efficiency (about 45\%), which is much lower than the linear alternator (about 90\%). However, it should be noted that the linear alternators are costly, which counteracts the advantages of thermoacoustic engines such as simplicity and low cost. Hence, there is a need to develop inexpensive linear alternators (e.g., loudspeaker) to facilitate the development of inexpensive and reliable thermoacoustic generators. A dditionally, the total thermal-to-electric efficiency of $10.9 \%$ is much higher than that 
of $2.4 \%$ for the thermoacoustic electric generator with loudspeaker because of reasonable design.

As shown in Fig. 3a, the length of the stub does not affect the transduction efficiency of the alternators, which is actually determined by the parameters of the al ternators as discussed in Section 2. When the load resistance is set as $10 \odot$, both two alternators have the same acoustic-to-electric efficiency of 0.445 . When the stub length $L_{s}=1.19 \mathrm{~m}$, the thermal-to-acoustic efficiency of both engine stages reaches the maximum, 0.412 and 0.343 , respectively, and the overall thermal-to-electric conversion efficiency reaches 0.109 .

It should be noted that the thermal-to-acoustic efficiency of TAE 1 is always higher than TAE2 as shown in Fig. 3d. This is due to two possible reasons. Firstly, as shown in Fig. 3c, the phase angle of TAE 1 is in the region of $-50^{\circ}<\varphi_{z}<-10^{\circ}$, while it is $-2^{\circ}<\varphi_{z}<30^{\circ}$ for TAE2. It is known that both the travelling and standing wave components can contribute useful thermodynamic conversion when the regenerator operates in the region with $-60^{\circ}<\varphi_{z}<0^{\circ}$, while the standing wave component does not contribute to the energy conversion when $0^{\circ}<\varphi_{z}<60^{\circ}[27-29,34]$. Secondly, the acoustic impedance is much higher at TAE 1 than TAE2. It is possible to increase the acoustic impedance at TAE2 to improve its thermal-to-acoustic efficiency, which decreases the efficiency at TAE 1 significantly and leads to a lower overall efficiency.

As shown in Fig. 3b, the acoustic power generation at two engine stages and the electric power generation at two alternators all increase significantly when the stub length increases from 1 to 1.5 meters, and then remain almost constant as the stub 
length further increases.

\section{Experimental setup}

A prototype has been constructed based on the proposed configuration and the optimized model. Fig. 4 is a photograph of the device. The dimensions of each component are summarized and listed in Table 2.

The through bore hole of the ball valve has the same inside diameter (i.e. $72 \mathrm{~mm}$ ) as the resonator pipe and a length of $225 \mathrm{~mm}$. The MAHE and SAHE are parallel-plate type heat exchangers which have the same dimensions and configuration as shown in our previous paper [30]. The regenerator is made of stainless-steel screen disks with the mesh number 120 and the wire diameter of 0.081 $\mathrm{mm}$. The calculated hydraulic radius is $0.0472 \mathrm{~mm}$.

The electrically heated hot heat exchanger $(\mathrm{HHE})$ is located below the regenerator. Part of the cross sectional area of $\mathrm{HHE}$ has a parallel plate structure, while the remaining part is solid metal with perpendicular holes for cartridge heaters as shown in reference [30].

In experiments, the acoustic pressure was measured and analyzed in real time by a computer. Twelve pressure transducers, labeled as $P_{1}$ to $P_{12}$ in Fig. 1 , are installed in the system to measure the distribution of the acoustic field. The pressure sensors are the piezoelectric type (CY-YD 208), supplied by Sinocera Piezotronics Inc., China. The data acquisition board is USB2831 supplied by Beijing Art Technology Development Co. Ltd., which has 12 bit precision and $250 \mathrm{kHz}$ sampling frequency. 
The output voltage from the piezoelectric transducer is amplified by a charge amplifier and then sent to the USB2831 board. Four calibrated NiCr-NiSi thermocouples, labeled as $T_{1 h}, T_{1 a}, T_{2 h}$ and $T_{2 a}$, are installed in the system as shown in Fig. 1. The thermocouple $T_{1 h}$ and $T_{2 h}$ are located at the hot end of the two regenerators, and $T_{1 a}$ and $T_{2 a}$ are located at the ambient end of the two regenerators. The thermocouples have an accuracy of $\pm 1^{\circ} \mathrm{C}$. Heating power is controlled by changing the supply voltage $(\mathrm{V})$ to the heaters and is displayed by a digital voltmeter. The current $(\mathrm{I})$ is measured by the ampere meter. The heating power can then be obtained as product of I and V. The electric output of the two alternators was measured by using a power meter, NA PUI PM 9800, with accuracy of $0.4 \%$ of the reading.

\section{Results and discussion}

In the experiments, pressurized helium at 1.8 M Pa was used as working gas. The working frequency was measured as $171 \mathrm{~Hz}$. During the experiments, the turning angle of the ball valve, load resistance of the alternator and the inputted heat power were adjusted to achieve high output electric power and efficiency. The experimental results are shown in Figs. 5, 6, 7 and 8.

The turning angle of the ball valve was initially set at 0 degrees (i.e. fully open). The load resistances of the two alternators were set at $10 \stackrel{0}{0}$. A fter turning the two electrical heaters on at $1.5 \mathrm{~kW}$, the thermoacoustic generator started the spontaneous acoustic oscillation when the temperatures reached $223{ }^{\circ} \mathrm{C}$ and $237{ }^{\circ} \mathrm{C}$ at the hot end of the regenerators of TAE1 and TAE2, respectively. The ball valve was then 
slightly turned to between $0^{0}$ (fully open) and $90^{\circ}$ (fully closed). Therefore, local acoustic resistance could be introduced to tune the acoustic field within the looped tube engine by setting the ball valve at a certain turning angle. Fig. 5 shows the effect of the turning angle of the ball valve on the electric power output of two alternators. The electric power outputted from the two alternators reaches a maximum when the turning angle is about $10^{\circ}$. In the experiments thereafter, the turning angle of ball valve is fixed at this optimal value.

Figs. 6 and 7 show the effect of the load resistance on the electric power output. The load resistance of one alternator is fixed as $10 \odot$, while that of the other changes from 2 to 50 @. From these two figures, it can be found that, the calculated and measured electric power output changes as the resistance increases in a similar fashion.

As the load resistance $R_{1, L}$ of Alternator1 increases, its electric power output changes significantly, and reaches a maximum when $R_{1, L}=10 \Omega$, while the electric output of A Iternator2 slightly decreases. The total electric power output from the two alternators reaches its maximum when $R_{1, L}=10 \Omega$.Similarly, as shown in Fig. 7 , the electric power output of A Iternator2 firstly increases and then decreases when its load resistance $R_{2, L}$ increases from 5 to $45 \Omega$ and reaches the maximum when $R_{2, L}=10 \Omega$. Differing from Fig. 6 , one can see that the electric power output from Alternator1 slightly increases as $R_{2, L}$ increases in this range. Similarly, the total electric power from the two alternators reaches its maximum when $R_{2, L}=10 \Omega$.

The turning angle of the ball valve was kept as $10^{\circ}$ and the load resistances of 
two alternators were both set as $10 \Omega$. Under such optimal operation conditions, the heating power input to each heater was adjusted 1.5 to $3 \mathrm{~kW}$ to investigate the influence of the heating power input, as shown in Figure 8. It should be noted that the heating power was always kept the same for two heaters so that the total heating power varied in between 3 to $6 \mathrm{~kW}$. As shown in Fig. 8, the overall thermal-to-electric conversion efficiency reaches its maximum $3.43 \%$ when the total heating power reaches $5.4 \mathrm{~kW}$ and the hot end temperature of the two regenerators reaches 512 and $452^{\circ} \mathrm{C}$, respectively. The total electric power output reaches its maximum $204 \mathrm{~W}$ when the total heating power reaches $6 \mathrm{~kW}$ and the hot end temperature of the two regenerators reaches 597 and $511^{\circ} \mathrm{C}$, respectively.

However, there is a discrepancy between the experimental results and the predictions by the model. There are several possible reasons: Firstly, the heat loss in experiments is large, which has not been taken in to account in the simulation. Secondly, the acoustic filed within such a loop-tube configuration is complicated and very sensitive to the dimensions of each component. There is a small discrepancy between the actual parts and the original design, which inevitably alters the acoustic fields within the actual engine. The ball valve has hel ped to partly correct the acoustic field, but more research work needs to be done to further understand the optimal acoustic field in such a complicated system. Thirdly, as the pressure amplitude is relatively higher, nonlinear acoustic behaviour exists in the tested rig. However, the DeltaEC is essentially a one dimensional linear thermoacoustic model that does not account for any nonlinear and two dimensional effects. Therefore, a more advanced 
theoretical model is required.

\section{Conclusions}

In this paper, a traveling-wave thermoacoustic electric generator with loudspeakers as alternators was designed, constructed and tested. It consisted of two thermoacoustic engine stages to convert thermal energy to acoustic power. Two loudspeakers were used as the alternators to extract and convert the engine's acoustic power to electricity. A new configuration was proposed to install these two alternators to the engine. One was installed within the engine loop which suppressed the Geoden acoustic streaming, while the other was installed at the end of a branched stub. In this way, the electric power output was significantly increased. A ball valve was used in the loop to partly correct the acoustic field that was altered by manufacturing errors. A prototype was built based on this new concept, which used pressurized helium at 1.8 MPa as the working gas and operated at a frequency of about $171 \mathrm{~Hz}$. In the experiment, a maximum electric power of $204 \mathrm{~W}$ when the hot end temperature of the two regenerators reaches $512{ }^{\circ} \mathrm{C}$ and $452{ }^{\circ} \mathrm{C}$, respectively. A maximum thermal-to-electric efficiency of $3.43 \%$ was achieved when the hot end temperature of the two regenerators reaches $597^{\circ} \mathrm{C}$ and $511^{\circ} \mathrm{C}$, respectively. The research results presented in this paper demonstrate that multi-stage travelling-wave thermoacoustic electricity generator has a great potential for developing inexpensive electric generators.

This research showed that the audio loudspeaker can produce over 100 Watts 
electricity without any modifications, which demonstrates the feasibility of developing inexpensive alternators based on audio loudspeaker technologies that are mature and widely available nowadays. Ultimately, this research shows the great potential of developing inexpensive thermoacoustic generators once such cheap alternators can be provided.

\section{Acknowledgement}

This work was supported by the Beijing Higher Education Young Elite Teacher Project (Grant No. Y ETP1172) and the National Natural Science Foundation of China (Grant N 0. 51006009).

\section{R eferences}

[1] Backhaus S, Swift GW . A thermoacoustic Stirling heat engine. Nature 1999; 399: $335-8$.

[2] Sun DM, Wang $K, X u Y$, Shen Q, Zhang XJ, Qiu LM. Thermoacoustic compression based on alternating to direct gas flow conversion. J Appl Phys 2012; 111: 094905.

[3] Yu Z, Jaworski AJ, Backhaus S. Travelling-wave thermoacoustic electricity generator using an ultra-compliant alternator for utilization of low-grade thermal energy. A ppl Energy 2012; 99: 135-45.

[4] Wu F, Chen L, Li D, Ding G, Zhang C, Kan X. Thermodynamic performance on a thermo-acoustic micro-cycle under the condition of weak gas degeneracy. A ppl 
Energy 2009; 86: 1119-23.

[5] Qiu LM, Lai BH, Li YF, Sun DM. Numerical simulation of the onset characteristics in a standing wave thermoacoustic engine based on thermodynamic analysis. Int J Heat M ass Trans 2012; 55: 2200-3.

[6] Swift GW. Thermoacoustics: A Unifying Perspective for Some Engines and Refrigerators. A coustical Society of A merica, New Y ork ,ISBN 0-7354-0065-2, 2002.

[7] Piccolo A. Optimization of thermoacoustic refrigerators using second law analysis. A ppl Energy 2013;103:358-67.

[8] Tijani MEH, Spoelstra S. A high performance thermoacoustic engine. J Appl Phys 2011; 110: 093519.

[9] Bailey P, Dadd M, Hill N, Cheuk C, Raab J, Tward E. High performance flight cryocooler compressor. In: Ross RG, editor. Cryocoolers 11, Springer US 2002; $169-74$.

[10]Redlich R. A summary of twenty years experience with linear motors and alternators. Linear Divers for Industry A pplications, M ay 31-J une 2. Nagasaki, J apan; 1995. <http://www.sunpower.com/library/pdf/publications/D oc0064.pdf>.

[11]Backhaus S, Tward E, Petach M. Traveling-wave thermoacoustic electric generator. A ppl Phys Lett 2004; 85: 1085-7.

[12] Petach M, Tward E, Backhaus S. Design and testing of a thermal to electric power converter based on thermoacoustic technology. In: 2nd International energy conversion engineering conference. The American Institute of 
A eronautics and A stronautics, Providence, R hode Island, A ugust 16-19; 2004.

[13]Oriti SM, Schifer NA. Recent Stirling conversion technology developments and operational measurements at NASA Glenn research center. 7th International energy conversion and engineering conference (IECEC 2009). Denver, CO, A ugust 2-5; 2009.

[14]Wu Z, Man M, Luo E, Dai W, Zhou Y. Experimental investigation of a $500 \mathrm{~W}$ traveling-wave thermoacoustic electricity generator. Chin Sci Bull 2011; 56: 1975-7.

[15]W u Z, Dai W, Man M, Luo E. A solar-powered traveling-wave thermoacoustic electricity generator. Sol Energy 2012; 86: 2376-82.

[16]Sun D, Wang $K$, Zhang $X$, Guo $Y, X u Y$, Qiu L. A traveling-wave thermoacoustic electric generator with a variable electric R-C load. Applied Energy 2013; 106: 377-382.

[17]Wu ZH, Yu GY, Zhang LM, Dai W, Luo EC. Development of a $3 \mathrm{~kW}$ double-acting thermoacoustic Stirling electric generator. A pplied Energy 2014.

[18]Y u Z, Jaworski A , Backhaus S. A low-cost electricity generator for rural areas using a travelling-wave looped-tube thermoacoustic engine. Proce Inst M ech Eng, Part A : J Pow Energy 2010; 224: 787-95.

[19]Y u Z, Saechan P, J aworski A J . A method of characterising performance of audio loudspeakers for linear alternator applications in low-cost thermoacoustic electricity generators. A ppl A coust 2011; 72(5): 260-7.

[20]Saha CR, Riley PH, Paul J, Y u Z, Jaworski A J, Johnson CM. Halbach array 
linear alternator for thermo-acoustic engine. Sensor A ctuator A: Phys 2012; 178: $179-87$.

[21]Y u Z, Jaworski AJ, Demonstrator of a combustion driven thermoacoustic electricity generator for remote and rural areas of developing countries. In: 19th International Congress on Sound and Vibration, 8-12 Jul 2012, Vilnius, Lithuania.

[22]Chen B, Yousif A, Riley P, Hann D. Development and Assessment of Thermoacoustic Generators Operating by Waste Heat from Cooking Stove. Engineering 2012; 4: 894-902.

[23]Ward W C, John C, Swift GW. Design environment for low-amplitude thermoacoustic energy conversion (DeltaEC). J ournal of the A coustical Society of A merica 1994; 95(6): 3671-3672.

[24]Belcher JR, Slaton WV, Raspet R, Working gases in thermoacoustic engines. J ournal of the A coustical Society of A merica 1999; 105: 2677-2684.

[25]Tijani MEH, Zeegers JCH, Waele TAM. Prandtl number and thermoacoustic refrigerators. Journal of the Acoustical Society of America 2002; 112(1): 134-143.

[26]A bdoulla K, K ang HF, Jaworski A J. Travelling-wave thermoacoustic electricity generator for rural areas using a side-branch alternator arrangement. Proceedings of the W orld Congress on Engineering 2013(W CE 2013), 1835-1840, London, United Kingdom, July 3 2013-J uly 52013.

[27] Kang HF, Li Q, Zhou G. Thermoacoustic effect of travelling-standing wave. 
Cryogenics 2009; 49: 112-119.

[28]Kang HF, Li Q, Zhou G. Heat driven thermoacoustic refrigerator based on travelling-standing wave. Energy Conversion and Management 2010; 51: 2103-2108.

[29] Kang HF, Li Q, Zhou G. Optimizing hydraulic radius and acoustic field of the thermoacoustic engine. Cryogenics 2010; 50: 450-458.

[30] Kang HF, Jiang $F$, Zheng HF, Jaworski AJ. Thermoacoustic travelling-wave cooler driven by a cascade thermoacoustic engine. A pplied Thermal Engineering 2013; 59: 223-231

[31]Sakamoto S, Imamura Y, and Watanabe Y . Improvement of Cooling Effect of Loop-Tube-Type Thermoacoustic Cooling System Applying Phase Adjuster. J apanese J ournal of A pplied Physics 2007; 46(7B): 4951-4955.

[32] Sakamoto S, Nishikawa M, Ishino1 T, W atanabe $Y$, and Senda J. Effect of Inner Diameter Change of Phase Adjuster on Heat-to-Sound Energy Conversion Efficiency in L oop-Tube-Type Thermoacoustic Prime M over. J apanese J ournal of A pplied Physics 2008; 47(5): 4223-4225.

[33] Sakamoto S, Sahashi K, Kitadani $Y$, Ishinol T, and Watanabe $Y$. A pplying Sub-L oop Tube to Control the Sound Field in a Loop-Tube-Type Thermoacoustic System. J apanese J ournal of A pplied Physics 2011; 50: 07HE 20.

[34]Zhou LH. Theoretical and experimental study on acoustic field modulation of regenerator in a thermoacoustic system. Dissertation. Technical Institute of Physics and Chemistry, Chinese A cademy of Sciences, B eijing, 2012. 